\title{
Pitfalls in the radiological diagnosis of inflammatory bowel disease
}

\author{
GP LEBRUN, MD, FRCPC
}

\begin{abstract}
Effective management of patients with inflammatory bowel disease (IBD) depends upon accurate diagnosis. The classic radiological features of this group of diseases have been extensively described. In addition, newer modalities such as ultrasound, computed tomography scanning, magnetic resonance imaging and nuclear medicine have been added to the diagnostic armamentarium. In spite of this the patient with IBD often presents a diagnostic dilemma as these conditions may mimic, and be mimicked by, other diseases. In particular, infectious, neoplastic and vascular etiologies should be considered in the differential diagnosis of IBD and suspected especially if the radiological appearances are not typical. Accurate diagnosis requires the selection of radiological examinations appropriate to the clinical situation which must be performed and interpreted with an awareness of the many pitfalls in this type of diagnosis. Can J Gastroenterol 1990;4(7):317-323
\end{abstract}

Key Words: Crohn's disease, Diagnosis, Radiology, Ulcerative colitis

\section{Ecueils du diagnostic radiologique des maladies inflammatoires de l'intestin}

RESUME: La prise en charge efficace des patients porteurs de maladies inflammatoires de l'intestin (MII) dépend de l'exactitude du diagnostic. Les caractéristiques radiologiques classiques de ce groupe d'affections ont été largement décrites. De plus, de nouvelles modalités font désormais partie de l'arsenal diagnostique - échographie, tomographie assistée par ordinateur, imagerie par résonance magnétique et médecine nucléaire. Malgré tout, le patient porteur de MII présente souvent un dilemne diagnostique vu que sa maladie peut en mimiquer une autre ou être mimiquée par elle. Des origines infectieuses, néoplasiques et vasculaires devraient être considérées lors du diagnostic différentiel des MII, surtout quand les caractéristiques radiologiques ne sont pas typiques. L'exactitude du diagnostic requiert la sélection d'examens radiologiques adaptés à l'état clinique; on doit les exécuter et interpréter en restant conscient des nombreux écueils que pose le diagnostic des MII.

Department of Radiology, Victoria General Hospital, Halifax, Nova Scotia

Correspondence and reprints: Dr GP LeBrun, Department of Radiology, Victoria General Hospital, 1278 Tower Road, Halifax, Nova Scotia B3H 2Y9. Telephone (902) 428-3770
$\mathrm{B}$ ECAUSE CROHN'S DISEASE AND ulcerative colitis are different, particularly with regard to treatment and response to therapy, effective management of patients with inflammatory bowel disease (IBD) depends upon accurate diagnosis of the basic disease and its complications.

Diagnostic imaging studies are important in the evaluation of patients with IBD at the time of initial diagnosis and for long term assessment. The role of radiology at the time of initial presentation is to detect the diseased segments, to document the gross pathological changes, to establish the correct diagnosis and to identify any complications. Because of the poor correlation between the clinical assessment of disease activity and radiological changes it is not necessary to perform studies on a routine periodic basis or during exacerbations without evidence of complications. Appropriate studies should be done while complications are suspected or as part of planning for surgery (1).

There are many difficulties in the radiological diagnostic process for IBD. In general terms these difficulties relate to the following: early changes may be subtle and nonspecific; Crohn's disease and ulcerative colitis may be difficult to 


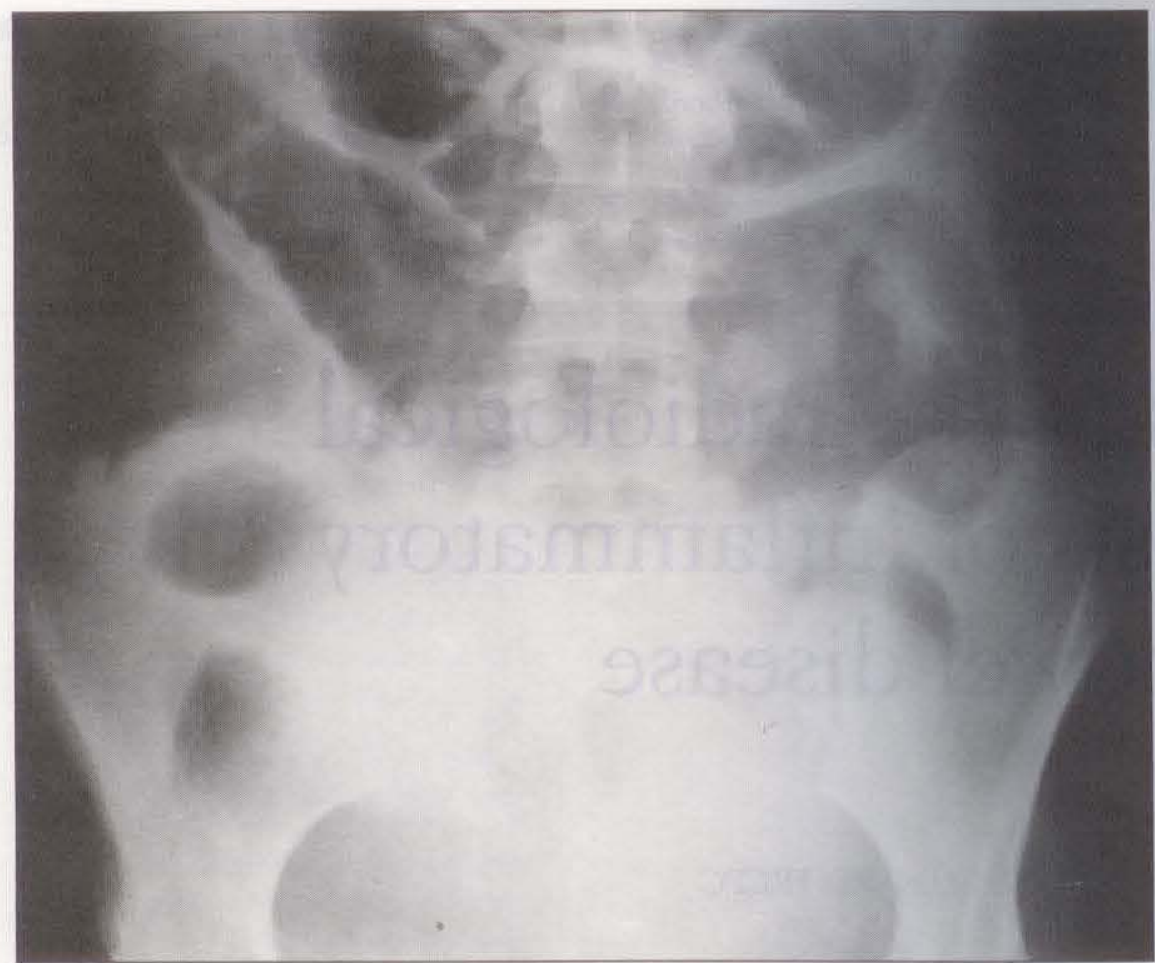

Figure 1) Plain film of the abdomen showing abnormal contour and polypoid mucosa in the transverse colon

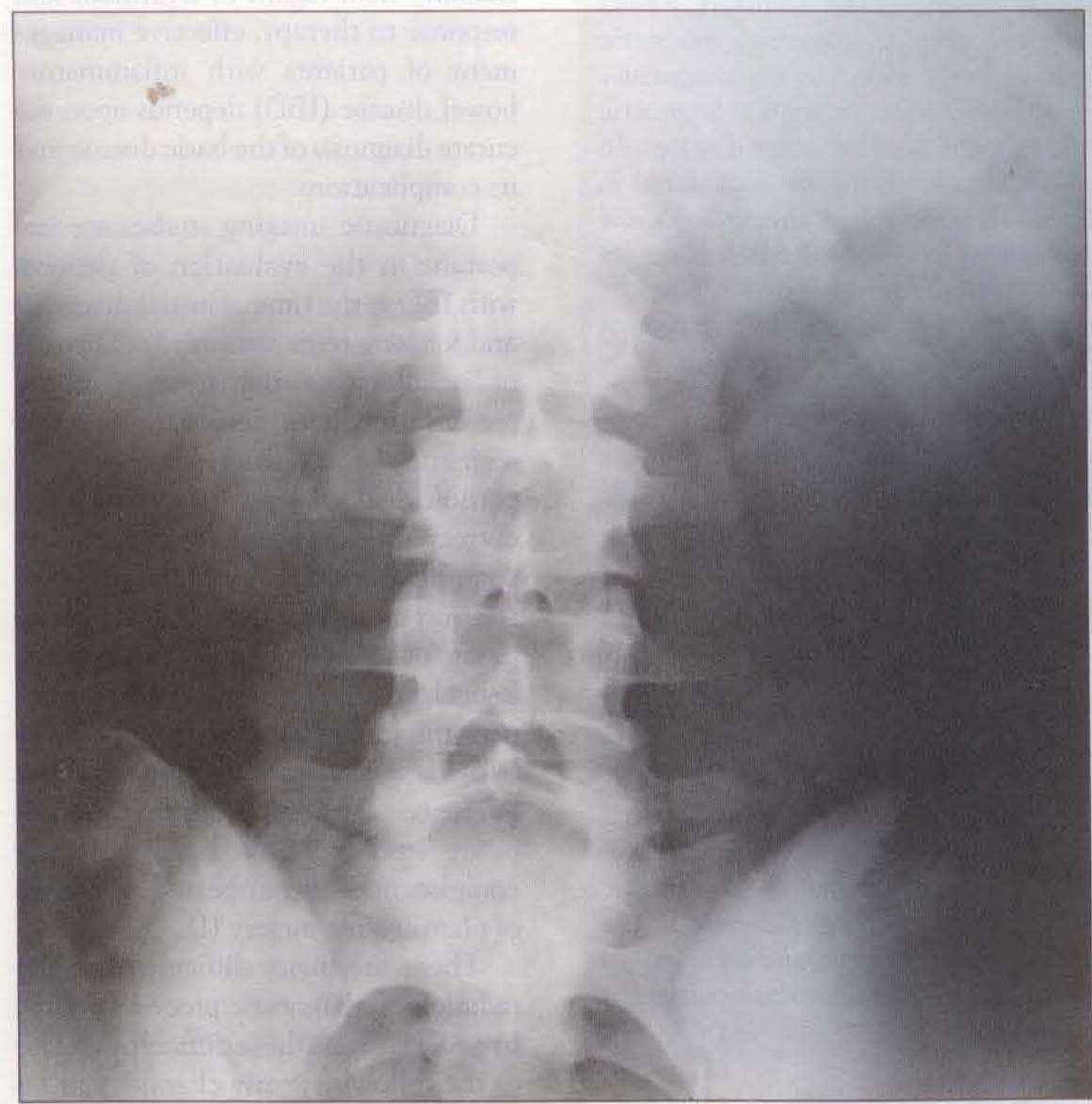

Figure 2) Marked mucosal edema resulting in 'thumb printing' in the transverse colon on abdominal plain film differentiate from each other; Crohn's disease and ulcerative colitis may mimic other diseases; other entities may mimic IBD; and complications may be difficult to diagnose or masked clinically by treatment.

This paper attempts to demonstrate some of these pitfalls by outlining the typical findings of IBD using various imaging methods and pointing out atypical patterns which should arouse suspicion. It is only through awareness of these atypical patterns that pitfalls or diagnostic errors can be avoided. It must also be stressed that any dis. crepancy between the clinical and radiological findings should be regarded as an important danger sign.

\section{PLAIN FILM DIAGNOSIS}

While the findings on plain abdominal films of patients with IBD are often nonspecific they may be very helpful in demonstrating the extent and severity of disease when considered in conjunction with clinical and endo. scopic findings. It is usually possible to avoid or postpone performing contrast studies in the acutely ill patient with IBD.

Bowel changes: The plain film changes of IBD are best seen in the colon which is normally outlined by gas but may also be seen in gas-filled loops of small bowel. In patients with insufficient naturally occurring gas, the colon can be safely outlined on plain films by rec. tally introduced air (2). The most common finding in involved loops of bowel is an alteration in contour as a result of mucosal edema. It may also be possible, in advanced cases, to visualize the ul. cerations and/or polyps that charac. terize the mucosal changes (Figures 1,2). Strictures and thickened bowel wall may occasionally be seen outlined by gas. The distribution of stool through the colon can also give an indication of the extent of involvement, as feces usually do not accumulate in diseased segments of bowel.

Toxic megacolon: This serious and potentially deadly complication occurs in both Crohn's disease and ulcerative colitis - it is more common in the latter. Mortality rates overall have been reported at 5 to $30 \%$ but if perforation 


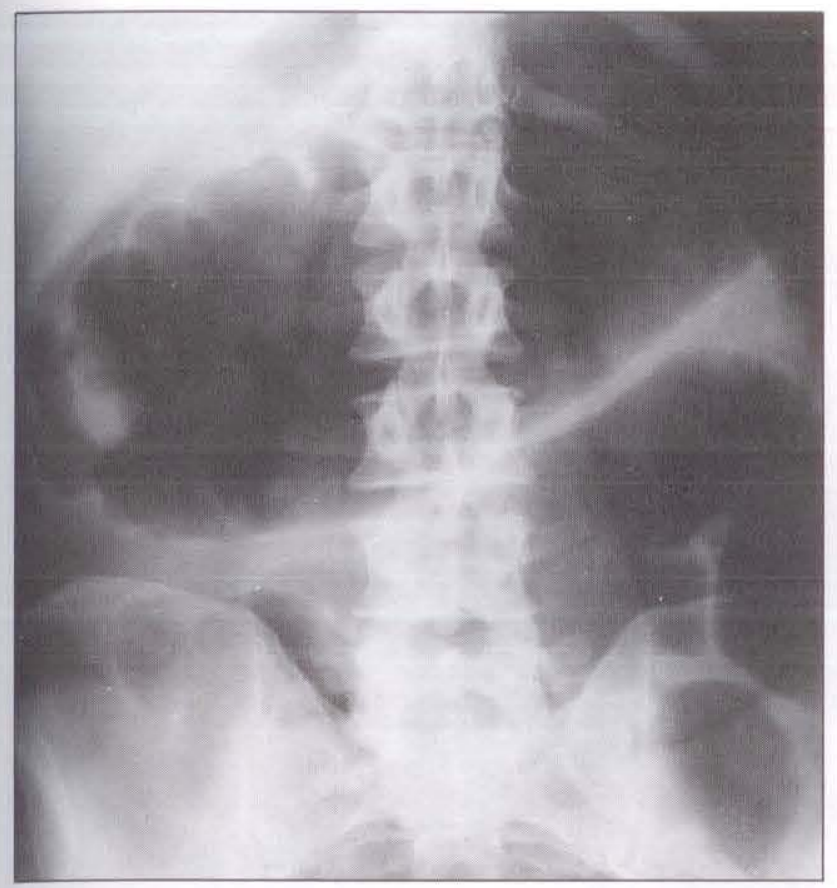

Figure 3) Abdominal plain film of a patient with toxic megacolon showing marked dilation and abnormal contour of the transverse colon

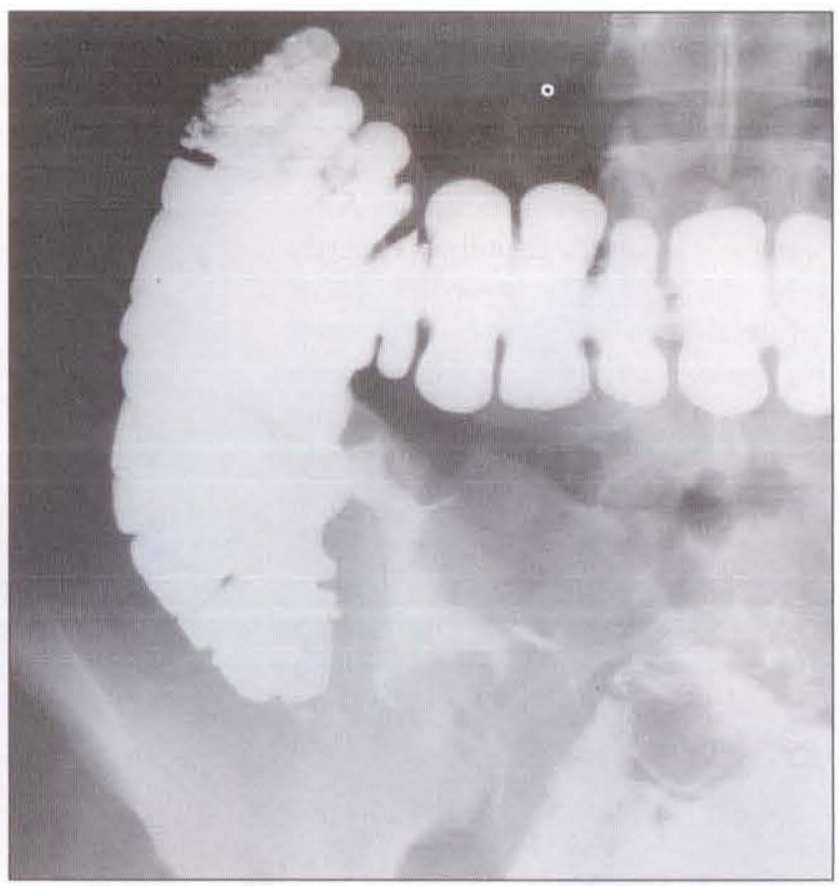

Figure 4) Lymphoma involving the terminal ileum. The mucosa is abnormal with nodular mass effects distorting and narrowing the lumen ensues mortality rises to 50 to $80 \%$. The key to diagnosis of this serious entity is a high clinical suspicion along with judicıous use of plain abdominal radiographs. Changes noted on sequential examinations are much more useful than a single examination. Radiological examinations are particularly important as treatment may mask the development of toxic megacolon. As noted, plain films can usually demonstrate the extent and severity of disease. The most prominent feature of toxic megacolon is dilation. This is most frequently seen in the transverse colon as it is the highest portion of the colon in the supine patient (Figure 3 ). The normal transverse colon measures less than $6 \mathrm{~cm}$ in diameter while in toxic megacolon it may measure up to $15 \mathrm{~cm}$. Dilation alone is not a diagnostic finding; the mucosal contour changes noted previously are very important in establishing the diagnosis. These include mucosal edema, nodules, thick bowel wall and occasional laceration. A small proportion of patients may have clinical evidence of toxic colon and radiographic evidence of mucosal abnormalities but no dilation.

These patients should be presumed to have toxic colon as perforation has been seen in the absence of dilation.

Contrast examinations have long been suspected as a precipitating factor in the development of toxic megacolon. Various studies have shown that 10 to $45 \%$ of patients with this entity have had a recent barium examination even though there is considerable variation in intervening time. Most patients are also on antidiarrheal drugs which have been implicated as a precipitating cause. While not proven as a cause of toxic megacolon the evidence warrants a cautious approach in the use of colonic barium studies in patients with an acute flare-up of IBD. They should only be used if plain films and prolonged small bowel series have not provided the necessary information, and should be performed using a minimum amount of barium and minimal distension.

\section{BARIUM STUDIES OF THE UPPER GASTROINTESTINAL TRACT}

While Crohn's disease primarily involves the small bowel and colon, the esophagus, stomach and duodenum are increasingly being recognized as being involved. The typical findings in the upper gastrointestinal tract are similar to those seen in the large and small bowel. The earliest finding is that of aphthous ulcers, the demonstration of which requires double contrast technique. The later changes of thickened or cobblestone mucosal folds and larger ulcers can be demonstrated with double or single contrast exams. While the changes of Crohn's disease in the upper gastrointestinal tract are relatively easy to demonstrate, the difficulty lies in the fact that they are nonspecific and are in fact more frequently the result of other etiologies.

Superficial erosions in the esophagus can be seen in other types of esophagitis such as herpetic and drug induced. The ulcers of reflux esophagitis, while usually more numerous and more distal in location, are often indistinguishable from Crohn's disease ulcers. Later changes of stricturing and rarely fistulas are also more commonly due to other types of esophagitis and tumours.

In the stomach, erosions similar to Crohn's disease are more commonly the result of erosive gastritis associated with alcohol ingestion, aspirin use, 


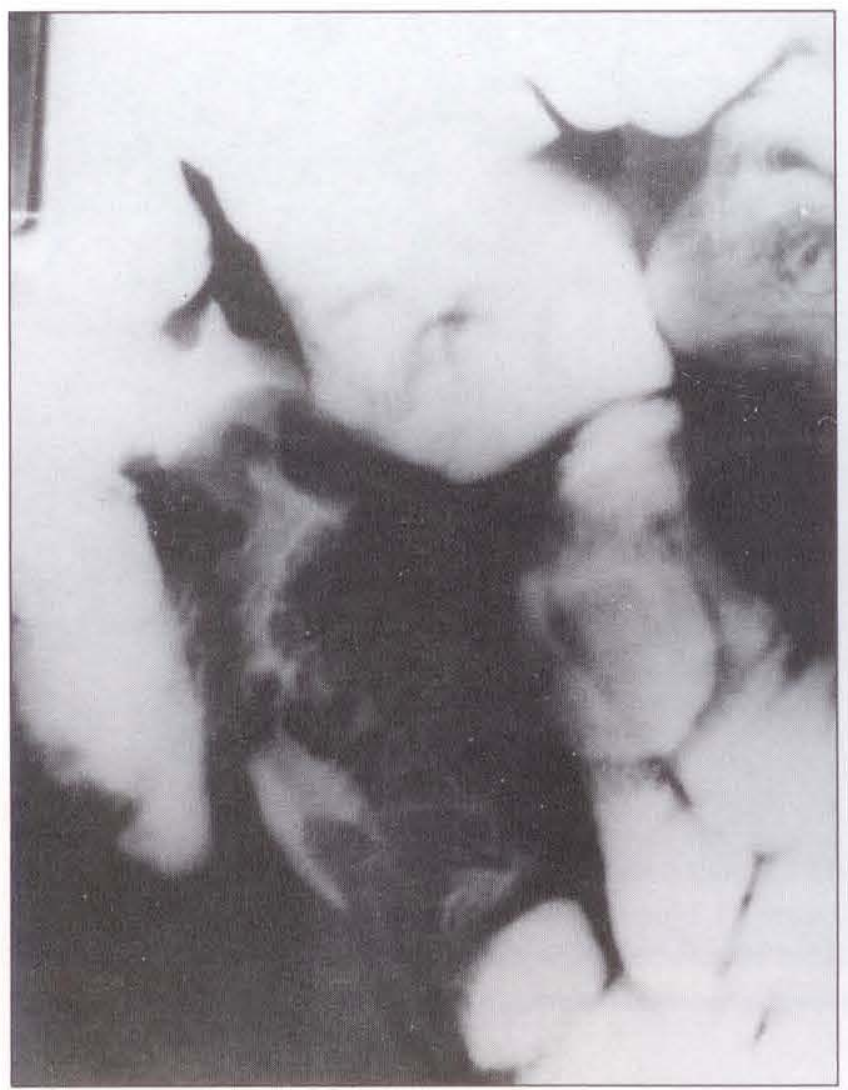

Figure 5) Deep underlying ulcer in the terminal ileum of a patient with Behçet's disease

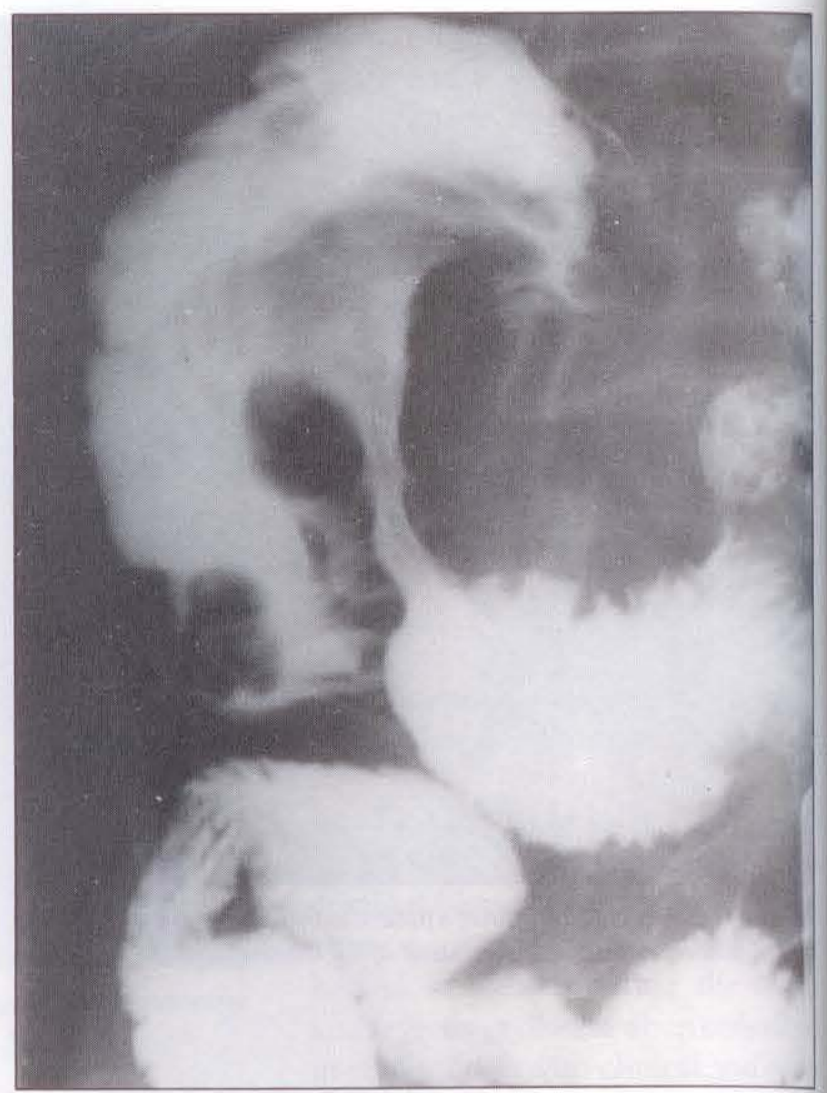

Figure 6) Carcinoid of terminal ileum with narrowing of the lumen. This patient was diagnosed nine years previously as having Crohn's disease of the ileum steroids, trauma and burns. The later scarring and stricturing involving primarily the antrum is also seen with peptic ulcer disease, corrosive gastritis, tumour, tuberculosis, syphilis and eosinophilic gastritis.

Erosions in the duodenum are seen in other types of duodenitis than Crohn's disease; thick duodenal folds are also more commonly seen in peptic duodenitis and secondary to pancreatitis. Strictures in the bulbar duodenum are a frequent result of peptic ulcer disease, and in the post bulbar duodenum strictures may be the result of adenocarcinoma.

While the radiographic changes of Crohn's disease in the upper gastrointestinal tract are nonspecific and are more frequently the result of other etiologies, the diagnosis should be suspected when these changes are found in patients with small bowel or colonic Crohn's disease. Confirmation of the diagnosis usually requires endoscopic evaluation and biopsy.

\section{BARIUM STUDIES OF THE SMALL BOWEL}

Technique: The traditional method of examining the small bowel is the follow-through study; it remains the routine examination in spite of claims of superiority for enteroclysis or small bowel enema. A well performed small bowel follow-through will be adequate for most cases provided the proper type and amount of barium are used with frequent filming and fluoroscopy with compression (3). The terminal ileum must be routinely examined fluoroscopically and with spot films. In order to assess the terminal ileum for superficial mucosal abnormalities and degree of distensibility, air can be introduced rectally when the ileum is opacified with orally ingested barium. This variation of the small bowel follow through is known as peroral pneumocolon (4). In the author's opinion, enteroclysis should be reserved for those cases not adequately assessed by routine followthrough examinations, augmented oc- casionally by peroral pneumocolon, as this will reduce the need for duodenal intubation and the increased expense and radiation associated with enteroclysis. Of greater importance than what type of examination is per. formed, however, is that an optimal study should be done using the tech. nique of the examiner's choice.

Typical findings: The classic findings of Crohn's disease involving the small bowel have been extensively described and are well known. As in other parts of the intestine, the earliest changes consist of superficial erosion and thick. ened folds. The mucosa may have a granular appearance on double contrast studies. Later changes include cobble. stone mucosa with deep fissures or ul. cers. The bowel wall is thickened with narrowing of the lumen and there is usually evidence of a mass effect result. ing from wall thickening, mesenteric changes and adenopathy. Sinus tracts and fistulas are a not infrequent finding in advanced disease. 


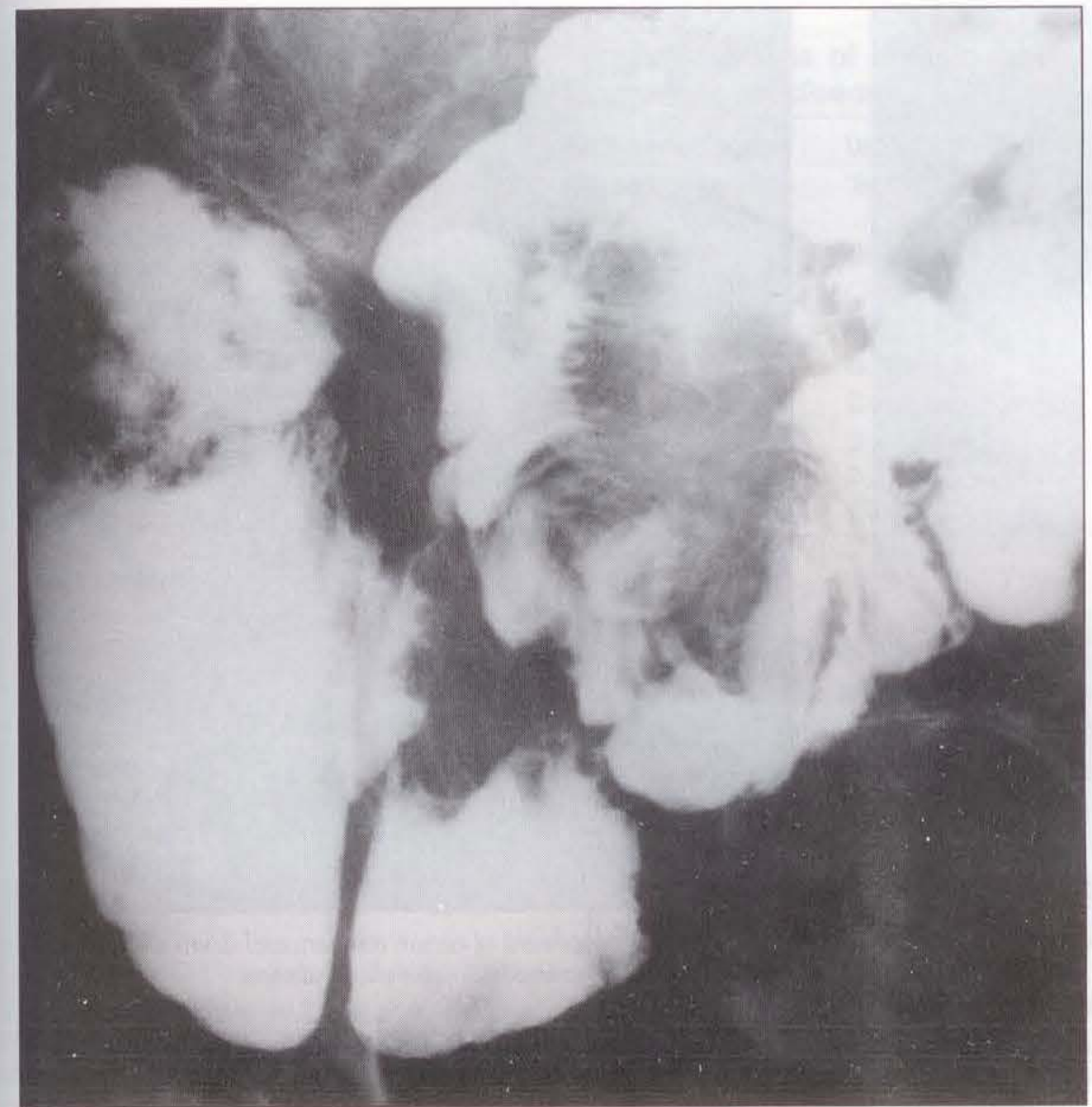

Atypical findings: The main difficulty in the initial radiological assessment of small bowel Crohn's disease is in establishing the correct diagnosis, as many other disease entities can cause similar changes, although they are less commonly encountered. In patients who show atypical findings or in whom the clinical and radiographic features do not agree, the possibility of another diagnosis should be considered. A list of entities which can simulate the various stages of Crohn's disease in the small bowel are outlined in Table 1. While many of these can simulate closely the changes of Crohn's disease, there are certain patterns which may be helpful in making the correct diagnosis.

The finding of diffuse inflammatory changes throughout the small bowel can be seen with Crohn's disease but should also raise the possibility an infectious process such as giardiasis or the uncommon eosinophilic gastroenteritis.

While Crohn's disease of the small bowel may spare the terminal ileum, this is uncommon and should raise suspicions about the diagnosis. Large

Fig ive 7) Distorted ileal loops in a patient with peritoneal carcinomatosis

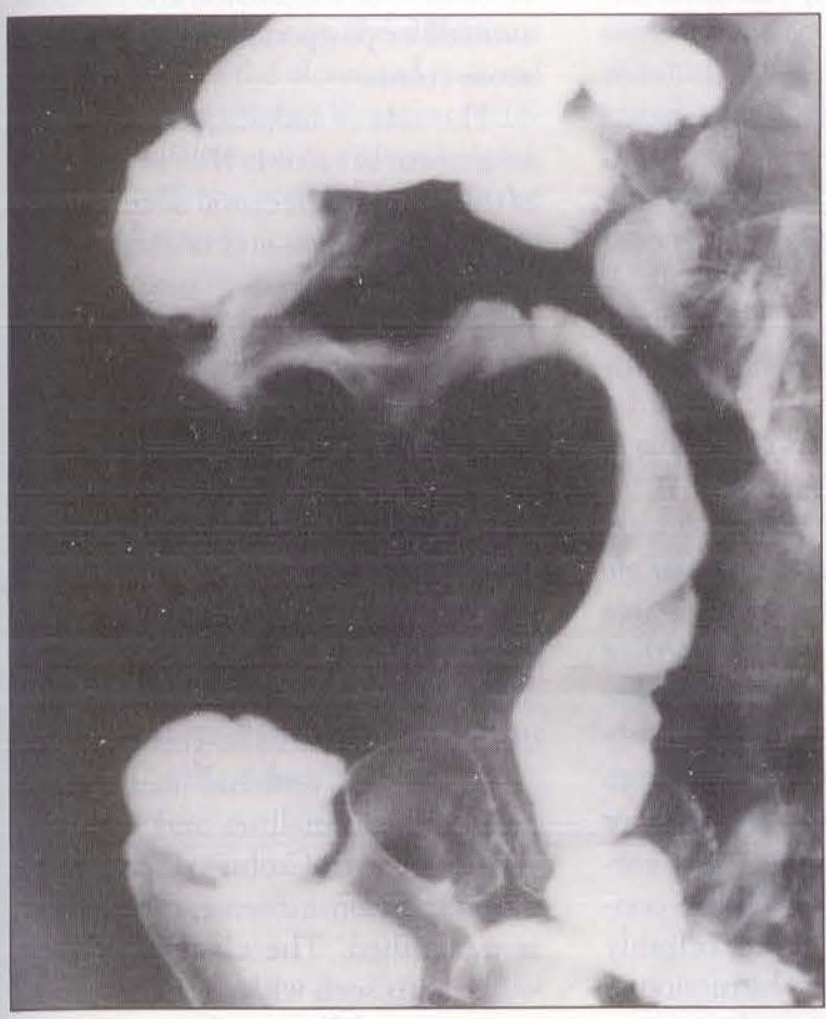

Figure 8) Narrowed and displaced terminal ileum due to direct invasion from an adenocarcinoma of the cecum

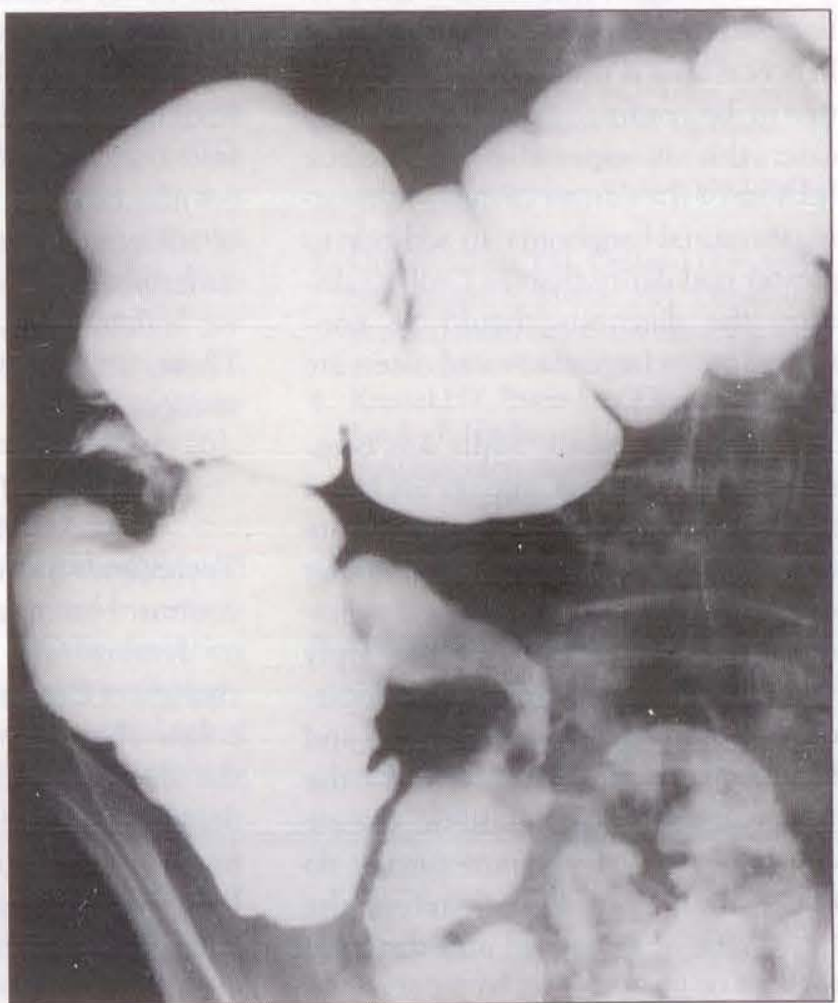

Figure 9) Metastatic colonic adenocarcinoma involving the terminal ileum. Note the primary lesion in the ascending colon 


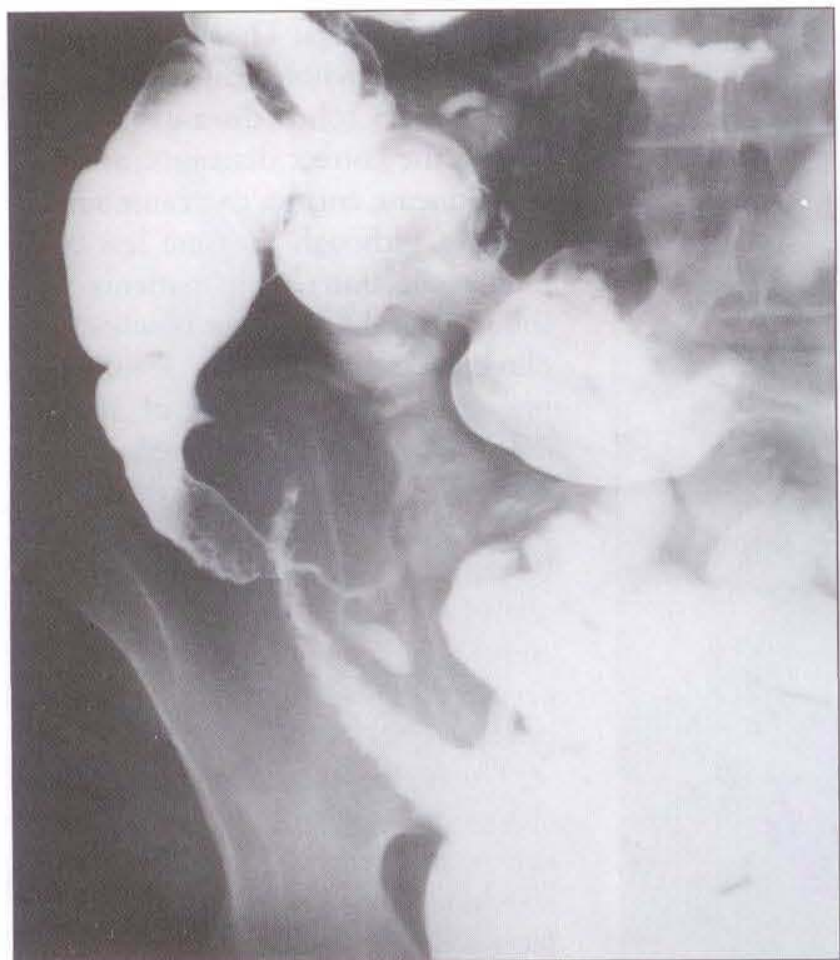

Figure 10) Narrowing of the terminal ileum and cecum with abnormal mucosa. This patient also had narrow segments in the transwerse and sigmoid colon. The findings are all due to metastatic adenocarcinoma probably arising in the colon

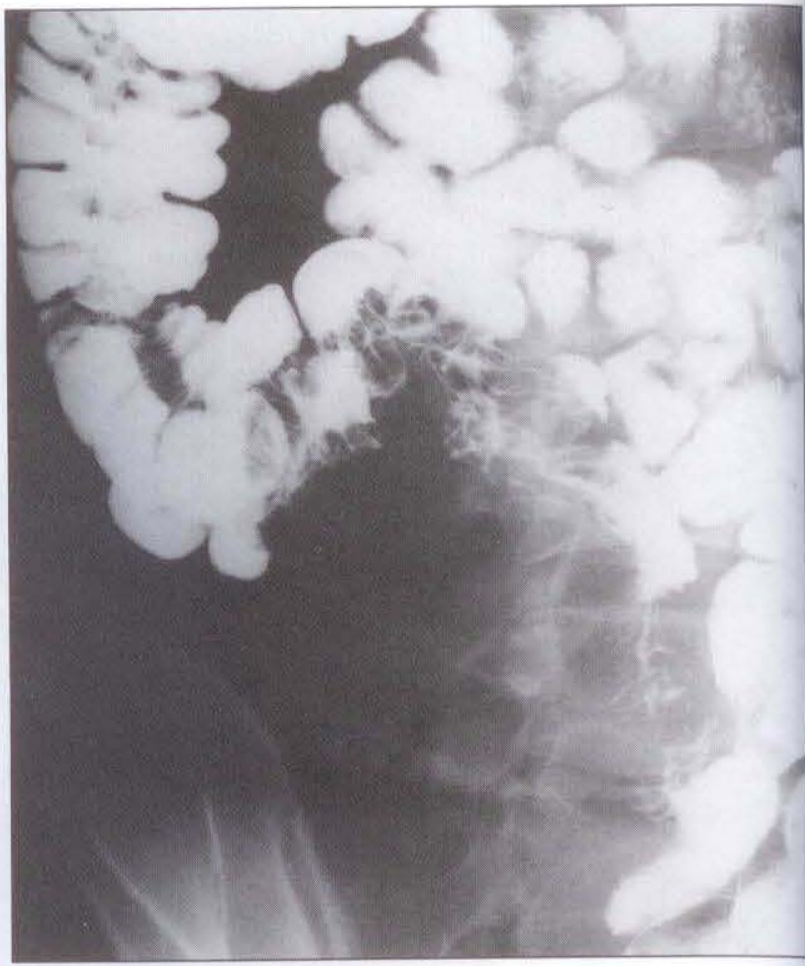

Figure 11) Displacement of cecum and terminal ileum with thickenet mucosal folds in a patient with appendiceal abscess discrete nodular masses especially in a segment of bowel without mucosal changes should suggest lymphoma (Figure 4). For practical purposes lymphoma in the ileocecal area is the most common entity to be confused with Crohn's disease; this is especially problematic because of the danger of delay in treating intestinal lymphoma. In addition to greater nodularity than in Crohn's disease, the diagnosis should be considered when large excavated ulcers are found (Figure 5).

Carcinoid tumour, while less common than lymphoma, also occurs in the terminal ileum and cecal area (Figure 6). This tumour also results in nodular masses distorting the bowel and sometimes resulting in the typical sharply angulated loops. Primary adenocarcinoma of the ileum is uncommon and has no particular predilection for the terminal segment. Metastatic disease and mesenchymal tumours usually do not arise in the mucosa, therefore, the bowel can be narrowed and distorted without or prior to involvement of the mucosa. In the gut, however, as elsewhere in the body, metastatic disease can have great variation in radiological appearance (Figures 6-10). Changes in the bowel secondary to vasculitis and ischemia may mimic IBD, but these also often spare the terminal ileum. Radiation enteritis is usually suspected from the clinical history. Finally, changes may be seen in the bowel as a result of inflammatory processes in adjacent structures such as appendicitis and pelvic inflammatory disease (Figure 11). These findings, however, are usually nonspecific.

\section{BARIUM STUDIES OF THE LARGE BOWEL}

Technique: Both double and single contrast barium examinations are able to demonstrate the well established changes of Crohn's disease or ulcerative colitis when properly performed. Only the double contrast study is able to demonstrate the aphthous ulcers or mucosal granularity which are the earliest abnormalities in IBD. Single contrast barium enema more reliably evaluates the presence of obstruction or fistulas. The choice of type of examination should be suited to the clinical situation. As mentioned earlier, colonic barium studies are contraindi. cated in toxic megacolon and should be avoided or postponed in patients with acute colitis.

The role of radiology in the initial assessment of colonic IBD as in the rest of the gut is to detect and document the anatomic changes and to establish the correct diagnosis. For practical purposes it is usually easy to establish the changes in the bowel as being due to IBD; the main difficulty encountered is differentiating Crohn's disease from ulcerative colitis. This differentiation is important because of the different prognoses and treatments. Many criteria have been identified, some of which are more reliable than others. The typical features of each of these diseases are outlined in Table 2 .

When the terminal ileum shows mucosal abnormalities and narrowing consistent with Crohn's disease in ad. dition to colonic disease, the diagnosis is established. The changes of back. wash ileitis seen with ulcerative colitis are quite different from those of Crohn's disease. Crohn's disease tends 


\begin{tabular}{l} 
TABLE 1 \\
Differential diagnosis of Crohn's dis- \\
ease of the small bowel \\
\hline Infections \\
Acute \\
Yersinia \\
Campylobacter \\
Giardia \\
Chronic \\
Tuberculosis \\
Actinomycosis \\
Histoplasmosis \\
Tumours \\
Lymphoma \\
Carcinoid \\
Adenocarcinoma \\
Other mesenchymal \\
Metastasis \\
Vascular/ischemic \\
Arteriosclerotic \\
Vasculitis \\
Bleeding disorder \\
Radiation \\
Endometriosis \\
Eosinophilic gastroenteritis \\
Adjacent inflammatory disease \\
Appendicitis \\
Pelvic abscess \\
\hline
\end{tabular}

to be right-sided and segmental whereas ulcerative colitis is always left-sided and continuous. Strictures and fistulas are hallmarks of Crohn's disease and are not seen with ulcerative colitis. The criteria which relate to the difference in appearance of the ulcers and mucosal polyps between the two diseases are difficult to evaluate and not often helpful. While in the majority of patients the correct diagnosis can be established using these criteria there remains a group of patients with pancolitis and normal small bowel, about $10 \%$ of the total, in whom the classification remains indeterminate.

The detection of a neoplasm occurring as a complication of colonic IBD has always been very difficult radiologically, with the tumour being advanced by the time of diagnosis. Endoscopy and biopsy have proven to be the method of choice for surveillance of complicating neoplasm in the colon.
TABLE 2

Typical features of colonic inflammatory bowel disease

\begin{tabular}{ll}
\hline Crohn's disease & Ulcerative colitis \\
\hline $\begin{array}{c}\text { Small bowel } \\
\text { involvement }\end{array}$ & 'Backwash ileitis' \\
Right side & Left side/rectal \\
Segmental & Continuous \\
Strictures & No strictures \\
Fistulae & No fistulae \\
Aphthous ulcers & Granular mucosa \\
Deep ulcers & Shallow ulcers \\
Cobblestone & Pseudopolyps \\
Toxic megacolon & Toxic megacolon \\
\hline
\end{tabular}

\section{CROSS SECTIONAL IMAGING}

In spite of the advent of the new modalities of ultrasound, computed tomography and magnetic resonance imaging, conventional barium studies remain the most accurate radiological method of assessing the mucosal and luminal changes of IBD. Plain films and barium studies, however, cannot reliably detect the associated abdominal changes that lie outside of the bowel itself, for example, abscess, phlegmon mesenteric changes and lymphadenopathy. It is particularly in the detection of these complications of IBD that ultrasound and computed tomography scanning are most valuable. Both computed tomography and ultrasound can show the bowel wall thickening of IBD, particularly in Crohn's disease. As it is often an early investigation in patients with abdominal problems, the diagnosis of IBD may be suggested on ultrasound (5). Computed tomography, while not the primary method of investigation, may show fistulous tracts (6).

\section{SUMMARY}

Diagnostic imaging studies play an important role in the initial investigation of patients with IBD by detecting and documenting the gross pathological changes. For follow-up, radiological studies are indicated when complica- tions are suspected or surgery planned. Plain films are often helpful in documenting changes in patients with acute colitis in conjunction with clinical and endoscopic changes, especially if toxic megacolon is suspected. Contrast studies should be avoided in these patients because of the possibility of precipitating toxic megacolon. The changes in upper gastrointestinal tract Crohn's disease are more commonly caused by other entities and will require endoscopy and biopsy for confirmation. The major difficulty in the radiological diagnosis of small bowel Crohn's disease is in differentiating it from the many 'lookalikes' that mimic its various findings. In the colon the main role of radiology initially is to differentiate between Crohn's disease and ulcerative colitis. Ultrasound, computed tomography scanning and possibly magnetic resonance imaging have assumed an important role in detecting abdominal changes that lie outside of the bowel, such as fluid collection and abscesses.

\section{REFERENCES}

1. Goldberg HI, Caruthers SB, Nelson JA, Singleton JW. Radiographic findings of the National Cooperative Crohn's Disease study. Gastroenterology 1979;77:925-37.

2. Bartram CI, Preston DM, LennardJones JE. The 'air enema' in acute colitis. Gastrointest Radiol 1983;8:615.

3. Ott DJ, Chen YM, Gelfand DW, van Swearingen F, Munitz HA. Detailed per-oral small bowel examination vs. enteroclysis. Radiology 1985;155:29-34.

4. Kressel HY, Evers KA, Glick SN, Laufer I, Herlinger $\mathrm{H}$. The peroral pneumocolon examination. Radiology 1982;144:414-6.

5. Kaftori JK, Pery M, Kleinhaus U. Ultrasonography in Crohn's disease. Gastrointest Radiol 1984;9:137-42.

6. Fishman EK, Wolf EJ, Jones B, Bayless TM, Siegelman SS. CT evaluation of Crohn's disease: Effect on patient management. Am J Roentgenol 1987;148:534-7. 


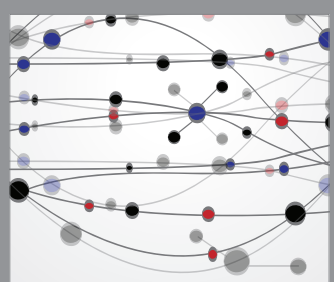

The Scientific World Journal
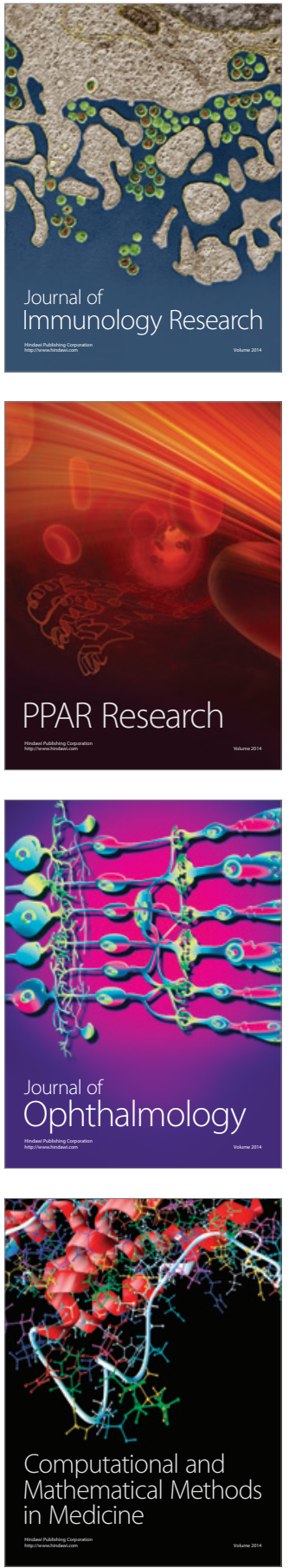

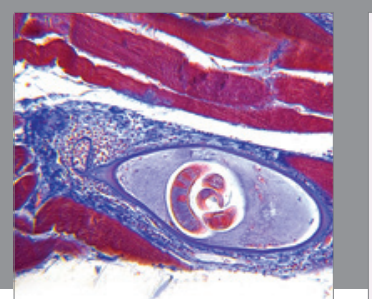

Gastroenterology Research and Practice

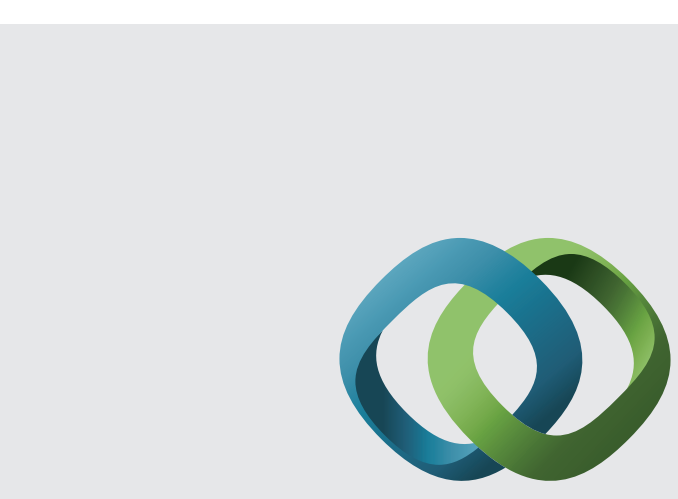

\section{Hindawi}

Submit your manuscripts at

http://www.hindawi.com
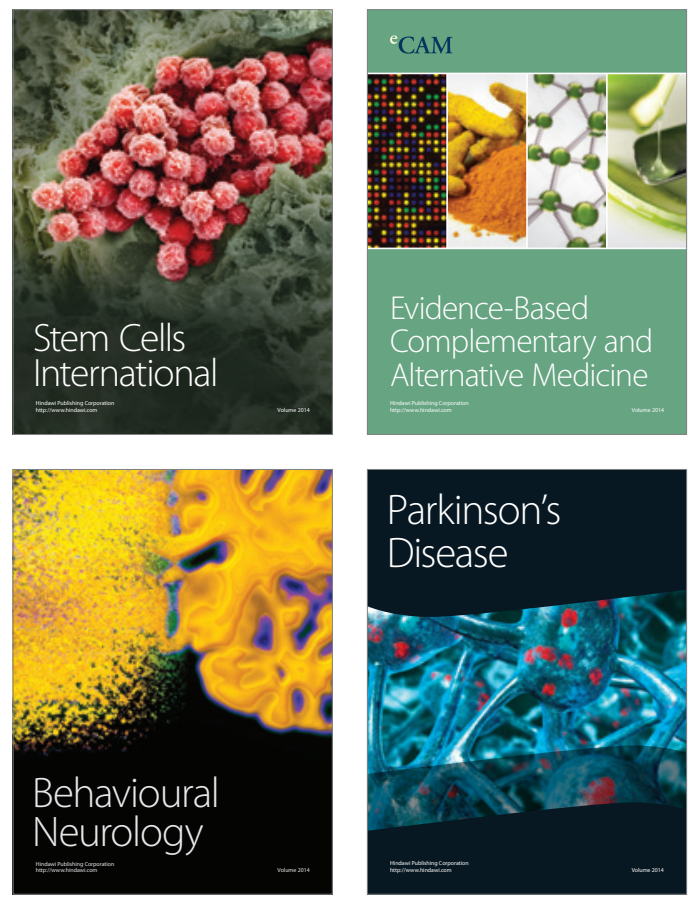
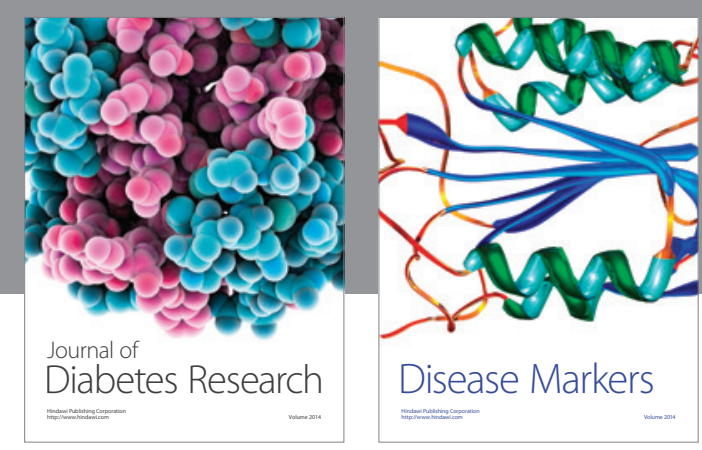

Disease Markers
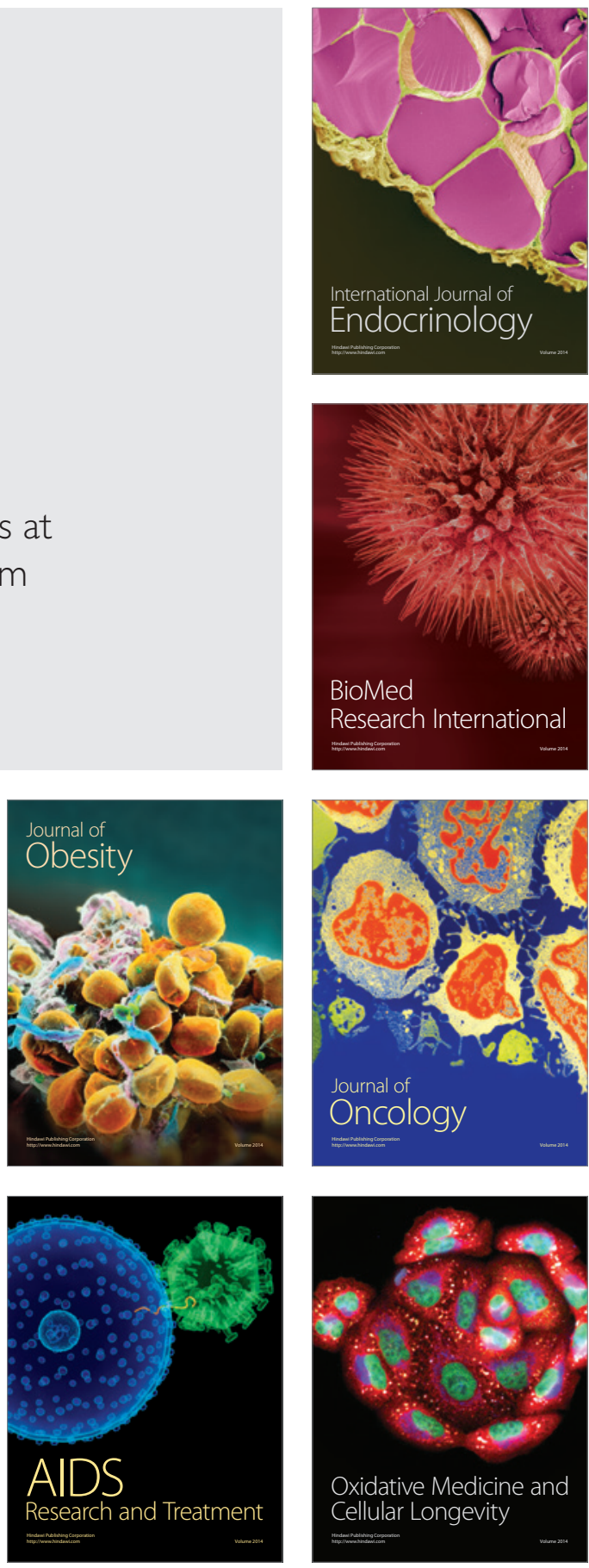